\title{
The effect of hygroscopicity on eddy covariance estimates of sea-spray aerosol fluxes: a comparison of high-rate and bulk correction methods
}

\author{
D. A. J. Sproson, I. M. Brooks, and S. J. Norris \\ Institute for Climate Atmospheric Science, School of Earth Environment, University of Leeds, UK \\ Correspondence to: D. A. J. Sproson (d.sproson@ see.leeds.ac.uk)
}

Received: 14 August 2012 - Published in Atmos. Meas. Tech. Discuss.: 3 September 2012

Revised: 28 November 2012 - Accepted: 22 December 2012 - Published: 13 February 2013

\begin{abstract}
The eddy covariance technique is the most direct of the methods that have been used to measure the flux of sea-spray aerosol between the ocean and atmosphere, but has been applied in only a handful of studies. However, unless the aerosol is dried before the eddy covariance measurements are made, the hygroscopic nature of sea-spray may combine with a relative humidity flux to result in a bias in the calculated aerosol flux. "Bulk" methods have been presented to account for this bias, however, they rely on assumptions of the shape of the aerosol spectra which may not be valid for near-surface measurements of sea-spray.

Here we describe a method of correcting aerosol spectra for relative humidity induced size variations at the high frequency $(10 \mathrm{~Hz})$ measurement timescale, where counting statistics are poor and the spectral shape cannot be well represented by a simple power law. Such a correction allows the effects of hygroscopicity and relative humidity flux on the aerosol flux to be explicitly evaluated and compared to the bulk corrections, both in their original form and once reformulated to better represent the measured mean aerosol spectra. In general, the bulk corrections - particularly when reformulated for the measured mean aerosol spectra - perform relatively well, producing flux corrections of the right sign and approximate magnitude. However, there are times when the bulk methods either significantly over- or underestimate the required flux correction. We conclude that, where possible, relative humidity corrections should be made at the measurement frequency.
\end{abstract}

\section{Introduction}

Sea-spray aerosol, generated in the open ocean through bubble bursting in whitecaps, or as droplets are physically ripped from the crests of waves by the wind, is the second largest natural source of aerosol into the atmosphere after dust (Hoppel et al., 2002). As such, sea spray is expected to have a major impact on global climate, both through direct (DossHammel et al., 2002) and indirect effects (Haywood et al., 1999). An understanding of production rates and transport of sea-spray is, thus, a prerequisite for an understanding of the climate system as a whole. Sea-spray is also a potentially important mediator of air-sea fluxes of heat, moisture and momentum at high wind speeds $\left(>25 \mathrm{~m} \mathrm{~s}^{-1}\right.$ ) (Andreas, 1992), where large volumes of re-entrant sea-spray may be produced.

Calculation of a sea-spray source function requires the quantification the flux of sea-spray aerosol into the atmosphere. Traditionally this is done through one of two methods. Firstly, under the assumption that the local production and deposition are equal, the flux of sea-spray aerosol into the atmosphere may be inferred through a model describing the dry deposition (i.e., the sink) of aerosol - the "equilibrium method" (Fairall et al., 1983; Fairall and Larsen, 1984; Smith et al., 1993; Hoppel et al., 2002). The second method relies on the assumption that spray production can be inferred from the fraction of the sea surface that is covered by whitecaps. The amount of spray ejected into the atmosphere per unit area whitecap is empirically derived through studies in the laboratory (Mårtensson et al., 2003), or at field sites generally located in the surf zone (Clarke et al., 2006). The fractional area of the ocean covered by whitecaps can then 
- again empirically - be related to the wind speed and seastate, allowing a wind dependent sea-spray source function to be derived (Monahan et al., 1982, 1986). Other methods used to calculate the surface source function, albeit less frequently, include the gradient method (Petelski and Piskozub, 2006); inverse modelling (Vignati et al., 2001; de Leeuw et al., 2003) and concentration increase with fetch (Reid et al., 2001).

Although the equilibrium and whitecap methods discussed above have both been widely used, they are both indirect, and source functions spanning up to 6 orders of magnitude have been reported (Andreas, 1998, 2002). More recent studies show source functions agreeing to within approximately one order of magnitude for particles with radii smaller than around $1 \mu \mathrm{m}$ (de Leeuw et al., 2011), although the equilibrium method is strictly only applicable to particles larger than about $3 \mu \mathrm{m}$ (Petelski and Piskozub, 2006; Andreas et al., 2010). A more direct method to measure the sea-spray source function is through the eddy covariance technique, whereby turbulent fluctuations in the vertical component of the wind are correlated with fluctuations in the aerosol spectrum to produce a net vertical transport of aerosol particles (Nilsson et al., 2001; Geever et al., 2005; de Leeuw et al., 2007; Norris et al., 2008, 2012). The derivation of a sea-spray source function using the eddy covariance method still, however, relies on some assumptions. Most importantly, what the eddy covariance technique actually measures is the net turbulent aerosol flux, therefore, it is only an adequate approximation to the true sea-spray source flux in conditions far from equilibrium. Note that this assumption is in direct contradiction of the conditions for which the equilibrium method is applicable, which relies on the assumption of zero net flux. In the limiting conditions of no deposition, or deposition equal to production, the eddy covariance or equilibrium method respectively give the best estimate of the surface source flux. However, neither of these methods can account for processes that occur below the measurement height, such as gas-toparticle conversion or coagulation of particles. In practice, however, where deposition generally is non-zero, but smaller than production, both methods will result in an underestimation of the true surface source flux. It is also possible for deposition to exceed the surface source, in which case the equilibrium method will overestimate the source flux and the measured net eddy covariance flux will be of the wrong sign. In either case, eddy covariance measurement of the net flux corrected for deposition is the most direct method to determine the surface source flux.

The calculation of an eddy covariance sea-spray source function requires the collocation of high-frequency aerosol and vertical wind measurements. This requires the use of a small, weatherproof aerosol spectrometer or a long sample line from the point of measurement back to the aerosol instrument. The use of such a sample line introduces a number of issues: a lag between wind and aerosol measurements; a damping of the aerosol fluctuations at high frequencies and a loss of particles to the walls of the sample line. Corrections for these effects exist, but may rely on unproven assumptions or become significant for larger particle sizes $(r>1 \mu \mathrm{m})$. Using a compact in situ instrument with a short sample line, however, makes drying the aerosol prior to measurement extremely difficult, thus, aerosol spectra will generally be recorded at ambient humidity.

Sea-spray aerosol is extremely hygroscopic and particles will rapidly grow or shrink in response to changes in the local relative humidity. In the presence of an upward relative humidity flux (which is almost ubiquitous in the marine environment), upward moving parcels of air $\left(w^{\prime}>0\right.$, where $w$ is the vertical velocity and the prime indicates a perturbation from the mean) will, on average, have a higher relative humidity than downward moving parcels. As sea-spray responds rapidly to RH fluctuations, a particle in an upward moving parcel may be larger than that in a downward moving parcel, even when these particles have equal dry radius. When measuring these aerosol in discrete size bins, this effect may cause the segregation of particles of equal dry radius into different bins, depending on the sign of $w^{\prime}$. When using the eddy covariance technique to determine aerosol flux, this will be interpreted as a net flux of aerosol, even if none exists. Fairall (1984), hereafter F84, and Kowalski (2001), hereafter K01, have both presented "bulk" methods of correcting for this apparent flux, based on mean meteorological conditions and assumptions of the shape of aerosol spectra within the marine boundary layer. Here, we describe a method of accounting for the humidity flux at the measurement timescale (a frequency of $10 \mathrm{~Hz}$ ) and compare this to the bulk corrections of both F84 and K01 for measurements from the SEASAW cruise in North Atlantic during March-April 2007. In the next section, we discuss the theory behind the bulk correction methods and describe the high-rate method we have used to account for humidity variance in the SEASAW data, give a brief overview of the SEASAW cruise, and processing that the data has undergone. In Sect. 3, we evaluate the validity of the assumptions of spectral shape with regard to the SEASAW data and present some alternative functional forms which better represent the aerosol spectra for these data. In Sects. 4 and 5, we compare the biases calculated through the high-rate method with those from the bulk methods, both in their original forms, and having been reformulated to use the functional forms which better represent the mean spectra recorded during SEASAW.

\section{Theory}

The hygroscopic nature of sea-spray aerosol means that the sizes of these particles change rapidly in response to changes in the ambient saturation ratio, $S$, defined as the ratio of ambient to saturation vapour pressure. In situations where there exists a vertical flux of the saturation ratio (i.e., $\overline{S^{\prime} w^{\prime}} \neq 0$, where the over-bar represents the averaging operator), the 
number of deliquescent particles in a certain particle size interval is a non-conservative scalar and, thus, size-segregated eddy correlation measurements of the number flux, $\overline{N^{\prime} w^{\prime}}$, where $N$ is the ambient aerosol concentration, may not be representative of the true aerosol flux, and net particle fluxes can be measured even where none are present.

\subsection{Bulk corrections}

Both F84 and K01 address the issue of apparent particle fluxes, both through an apparent transfer velocity, $\Delta v_{\mathrm{d}}$, which is the transfer/deposition velocity induced through the vertical flux of saturation ratio. Both give the dependence of aerosol radius on humidity as

$r(S)=r_{0}\left[1+\frac{\gamma}{1-S}\right]^{\frac{1}{3}}$,

where $r_{0}$ is the dry aerosol radius and $\gamma$ is a parameter related to the aerosol chemistry, taken as unity by F84 for clean marine air. Taking the derivative of Eq. (1) with respect to $S$, we have

$\frac{\partial \bar{r}}{\partial \bar{S}}=\frac{\gamma \bar{r}}{3(1-\bar{S})^{2}+3 \gamma(1-\bar{S})}$.

Note: we have substituted $\bar{r}_{0}=\bar{r}[1+\gamma /(1-\bar{S})]^{-1 / 3}$ in the above.

Under a fluctuation in saturation ratio, $S^{\prime}$, the change in the ambient concentration at a fixed radius is a combination of the translation of the particle distribution in radial space and a "renormalisation" which occurs due to the change in the size intervals over which the distribution is defined. These two effects result in an induced change in the particle distribution which can be written, following K01, as

$N^{\prime}=r^{\prime}\left[\frac{\partial \bar{N}}{\partial \bar{r}}+\frac{\bar{N}}{\bar{r}}\right]$.

Both K01 and F84 make the assumption that the aerosol spectrum can be approximated through a Junge power law (Junge, 1963) of the form

$\bar{N}=\alpha \bar{r}^{-(\beta+1)}$,

where $\beta$ is typically about 3 and, thus

$\frac{N^{\prime}}{\bar{N}}=-\beta \frac{r^{\prime}}{\bar{r}}$.

Combining Eqs. (2) and (4) with the definition of the deposition velocity and taking $\frac{\partial \bar{r}}{\partial \bar{S}} \equiv \frac{r^{\prime}}{S^{\prime}}$, we arrive at K01's expression for the bias velocity due to humidity fluctuations and hygroscopicity:

$\Delta v_{\mathrm{d}}=\frac{-\gamma \beta}{3(1-\bar{S})^{2}+3 \gamma(1-\bar{S})} \overline{w^{\prime} S^{\prime}}$.
K01 reformulates Eq. (5) in terms of temperature and moisture fluxes, however, this is not required here. F84 makes the further approximation that, in the surface layer,

$\overline{w^{\prime} S^{\prime}}=c_{\mathrm{d}}^{\frac{1}{2}} u_{*}(1-\bar{S})$,

resulting in F84's expression for the bias velocity:

$\Delta v_{\mathrm{d}}=\frac{\gamma \beta}{3(1+\gamma-\bar{S})} c_{\mathrm{d}}^{\frac{1}{2}} u_{*}$.

Here, $c_{\mathrm{d}}$ is the drag coefficient which can be calculated from turbulence data or approximated through empirical relationships (Large and Pond, 1981; Yelland et al., 1998) and $u_{*}$ is the friction velocity.

Both of these methods provide a bulk means of estimating the bias velocity due to hygroscopicity, but both rely on the assumption that aerosol spectra can be well represented with the use of a Junge power law, and F84's equation relies on the accuracy of the approximation of $\overline{w^{\prime} S^{\prime}}$.

\subsection{High-rate corrections}

The collocation of high frequency measurements of humidity and size-resolved aerosol spectra during SEASAW allows the effect of hygroscopicity to be explicitly calculated. In order to do this, the $10 \mathrm{~Hz}$ CLASP spectra must be individually corrected to a reference humidity, typically the run-mean relative humidity, following Zhang et al. (2006) and Lewis and Schwartz (2003).

Such a correction is relatively simple in the mean sense, where a long averaging period means that all size channels will have adequate counting statistics and a simple functional form (for example, a Junge power law) can be fitted to the mean spectrum. Under a humidity correction, channel boundaries will change, but the number of particles, $N$, in each channel will be unchanged. Using the new channel boundaries, $\mathrm{d} N / \mathrm{d} r$ and a new mean radius for each channel can easily be calculated. If the spectrum is required over specific channel boundaries or at a specific radius, this can easily achieved by using the fit to the adjusted spectrum as an interpolant.

However, when adjusting spectra measured over much shorter time periods (here measurements are at $10 \mathrm{~Hz}$ ), things are not so simple. Following an adjustment for relative humidity, each individual spectrum will be defined over a different set of channel limits. Additionally, the counting statistics for a spectrum measured over such a short period of time will be much noisier than for a mean spectrum, and indeed may show little or no resemblance to the mean case. This makes it both difficult and inappropriate to use a functional fit to the spectra to redefine them over a consistent set of channel boundaries, and we must find an alternative means of evaluating these adjusted spectra over consistent limits. This is achieved as follows. Each $10 \mathrm{~Hz}$ spectrum is adjusted from ambient humidity to the run-mean humidity. A log-linear fit 
of the form $\mathrm{d} \mathfrak{N}_{i}=a_{i} r^{b_{i}}$ is then calculated for each channel in the adjusted spectrum, based on the values of $\mathrm{d} N / \mathrm{d} r$ in that channel and those in any neighbouring channels. This fit provides an estimate of the particle distribution across each channel, as the significant gradient in concentration with size applies within as well as between channels, and needs accounting for in the correction procedure. Integrating each channel's fit across the width of the channel then gives an estimate of the total number of particles, $\mathcal{N}_{i}=\int_{r_{i}} \mathrm{~d} \mathfrak{N}_{i} \mathrm{~d} r$, in that channel. This approximation is subject to some error, which we define as $\epsilon_{i}=\mathcal{N}_{i}-N_{i}$, where $N_{i}$ is the true particle number in the channel. We then calculate the overlap between the boundaries for each CLASP channel $j$ at the ambient humidity and each CLASP channel $i$ at the run-mean humidity. For each case where an overlap is present, we define the interval of the overlap as $\left[a_{i, j}, b_{i, j}\right]$. The number of particles which move from channel $j$ to channel $i$ due to the shift in channel boundaries under the humidity adjustment is then

$$
\Delta N_{i, j}=\left(1+\frac{\epsilon_{i}}{N_{i}}\right) \int_{a_{i, j}}^{b_{i, j}} \mathrm{~d} \mathfrak{N}_{i} \mathrm{~d} r,
$$

where the factor of $\epsilon_{i} / N_{i}$ is introduced to account for the error introduced in assuming a log-linear aerosol distribution through each CLASP channel. Note that this gives identical results to solving for $a_{i}$ and $b_{i}$ so that $\int_{r_{i}} \mathrm{~d} \mathfrak{N}_{i} \mathrm{~d} r=N_{i}$, however, it is significantly cheaper computationally.

The individual $10 \mathrm{~Hz}$ aerosol spectra from a 20-min data record are very variable, particularly in the larger CLASP size channels, and most have a spectral shape very different to that of the mean spectrum (Fig. 1). This is due to the discrete nature of aerosol measurements and the relatively small volume $(5 \mathrm{~mL})$ of each $10 \mathrm{~Hz}$ measurement. The black line in Fig. 1 shows this mean spectra once it has been corrected from the run-mean relative humidity $(60 \%)$ to a reference humidity of $80 \%$. The blue line in Fig. 1 shows the mean of the $1200010 \mathrm{~Hz}$ spectra once they have been individually corrected to $80 \%$ relative humidity via a $10 \mathrm{~Hz}$ humidity measurement collocated with the CLASP instrument. Note that after this procedure, each of these spectra is defined over a different set of channel limits, depending on the sign and magnitude of the individual relative humidity adjustment. This mean spectrum is, thus, defined over the mean channel limits of the high-rate aerosol spectra. While this is not useful for any analysis, it does show that before rebinning, correcting for humidity before time-averaging produces an almost identical spectrum to that produced by humidity correcting a run-mean aerosol spectrum. The dashed red line in Fig. 1 shows the mean of the high-rate spectra after they have been both corrected for relative humidity fluctuations and re-binned back to the original CLASP size channels at $10 \mathrm{~Hz}$. This mean spectrum is almost identical to the other two mean adjusted spectra, showing that

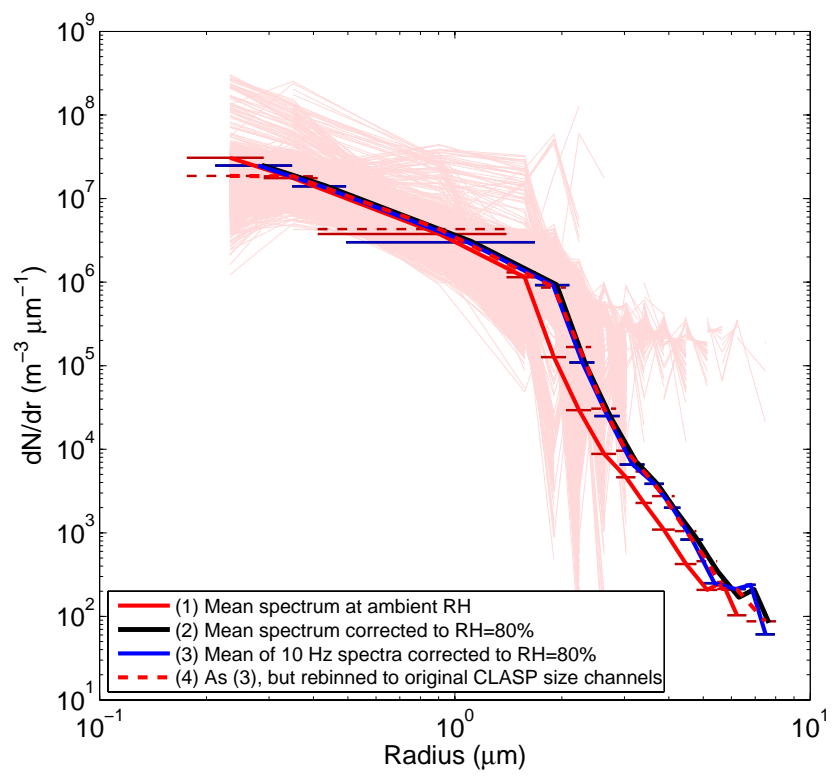

Fig. 1. An example of using the high-rate relative humidity correction technique described here. The pale red lines show the 12000 individual spectra which were measured at $10 \mathrm{~Hz}$ by the CLASP unit. The solid red line show the mean of these spectra at the mean ambient relative humidity. The black line shows the mean spectra corrected from the mean relative humidity $(60 \%)$ to a reference humidity of $80 \%$. The blue line shows the mean of the 12000 individual spectra after each has been adjusted to $80 \%$ relative humidity (note that this spectrum is then defined over the mean channel boundaries of these individual adjusted spectra). The dashed red line shows the mean of the 12000 spectra once they have been both adjusted to $80 \%$ and re-binned back to the original CLASP channel limits.

our re-binning technique works well at $10 \mathrm{~Hz}$, where spectral shapes are very variable, and many spectra include isolated channels in which no aerosol particles were recorded. The only difference between this spectrum and the other two adjusted mean spectra is that the local maximum in channel 13 has been smoothed out during the re-binning, and channel 1 has a significantly smaller particle count than either of the other adjusted mean spectra. This latter effect is due to the nature of the re-binning, where individual particles are moved from one channel into another. When correcting from a low relative humidity to a higher, as in this case, particles will generally be moved from smaller bins to larger (as particle size increases with increasing relative humidity), however, we have no information about the aerosol spectra for particle sizes smaller than the lower limit of CLASP channel 1. This means that particles will move out of this channel into channel 2, but none will move into it. The number of particles in channel 1 after adjustment will thus be erroneously small, and we must reject this channel from any further analysis. Equally, when adjusting from a higher relative humidity to a lower, we cannot ascertain the number of large particles which would move into the largest measurement bin, thus, 
under such an adjustment the largest measurement bin would have to be dropped. Note that adjusting relative humidity to a reference value of $80 \%$ is a rather stringent test of the humidity correction algorithm. In practice, if the particle flux in each channel is the same sign, the $10 \mathrm{~Hz}$ spectra need only be adjusted to the run-mean relative humidity for the purposes of evaluating the flux bias for a single record. The mean flux spectra may then later be adjusted to $80 \%$ humidity (or any other reference value) using standard approaches. In this case, measurements will be corrected to both lower and higher relative humidities, and both the smallest and largest measurement bins must be disregarded. If the particle flux changes sign between size bins, adjustment of the flux spectrum may be inappropriate and a high-rate correction to the reference humidity required. In this case, channels must be rejected depending on the largest $\mathrm{RH}$ adjustment required in this process. It should be noted that, contrary to traditional rebinning methods, which rely on a smooth aerosol spectrum, the high-rate rebinning technique can maintain the spectral shape of a noisy $10 \mathrm{~Hz}$ sample (Fig. 2).

\subsection{SEASAW Data}

The data which are used to test the high rate humidity corrections are from cruise D317 (21 March-12 April 2007) of the RRS Discovery made as part of the SEASAW project, a UK contribution to the international SOLAS programme (Brooks et al., 2009; Norris et al., 2012). Three dimensional winds and sonic temperature are available at $20 \mathrm{~Hz}$ from a Gill sonic anemometer, pressure and water vapour density are available, also at $20 \mathrm{~Hz}$, from a LI-COR LI-7500 open path gas analyser. Size resolved aerosol spectra are recorded at $10 \mathrm{~Hz}$ in 16 unequally spaced channels ranging between radii of 0.18 and $8.88 \mu \mathrm{m}$ by a CLASP instrument (Hill et al., 2008).

Aerosol, turbulent winds and humidity are time-matched at $10 \mathrm{~Hz}$ and split into "runs" of $20 \mathrm{~min}$. These runs are checked to ensure that sonic temperature and momentum flux ogives and relative humidity flux ogives are suitably well behaved, with a characteristic flattening of the curve at low and high frequencies and a minimal distortion at the wave scale (Fairall et al., 1997). Any runs, where this was not the case, were rejected from the analysis. The run-mean $10 \mathrm{~Hz}$ relative humidity timeseries was also required to lie within $\pm 10 \%$ of the low-frequency relative humidity from a Vaisala HMP45 humidity probe (part of the ship's permanent surface meteorology instrumentation) and all runs, where this was the case, were visually examined to ensure that the LI-COR and low-frequency relative humidity timeseries showed a similar signal. Once this quality control had been carried out, runmean CLASP aerosol spectra were then visually examined to remove any spectra that were clearly contaminated. This quality control left a total of 12420 -min runs with which to perform the analysis.

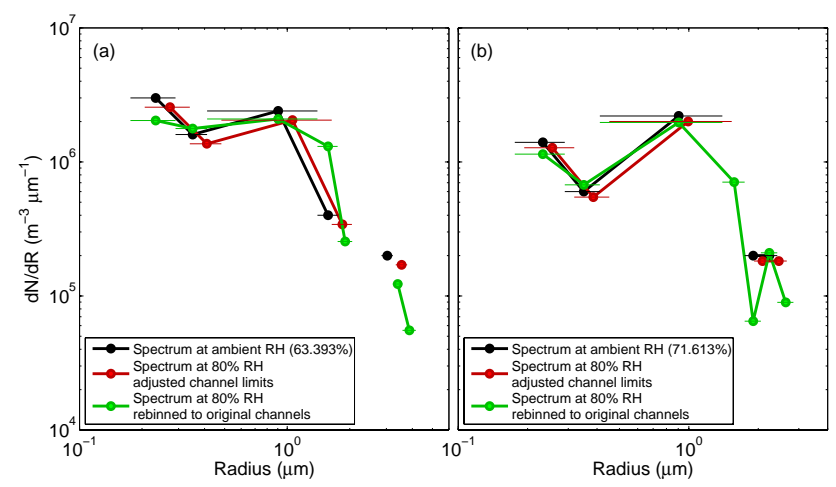

Fig. 2. Two examples of a relative humidity correction applied to sea-spray aerosol spectra recorded at $10 \mathrm{~Hz}$. The black lines show the original spectra at ambient humidity, the red lines show these spectra corrected to $80 \%$ relative humidity, and the green lines these corrected spectra after re-binning to the original instrument channels.

\section{Functional fits to SEASAW data}

Both F84 and K01 assume that, in the mean, an aerosol spectrum can be well described by a Junge relation of the form $N=\alpha r^{-(\beta+1)}$, with $\beta$ typically around 3. However, it is not clear that such a relationship is appropriate for the SEASAW measured aerosol spectra, which tend to have a smaller change in $\mathrm{d} N / \mathrm{d} r$ (in log space) with radius at smaller values of $r$, with a distinct change in gradient at around $r=1.5-$ $2 \mu \mathrm{m}$, between CLASP channels 4 and 5 (Fig. 3). The green lines in Fig. 3 show Junge fits which are calculated by substituting $N^{*}=\log (\mathrm{d} N / \mathrm{d} r)$ and $r^{*}=\log (r)$ and finding a linear least-squares fit to $N^{*}$ and $r^{*}$. This leaves us with $N^{*}=b r^{*}+a$ and, thus

$$
\begin{aligned}
\frac{\mathrm{d} N}{\mathrm{~d} r} & =\exp (b \log (r)+a) \\
& =e^{a} r^{b},
\end{aligned}
$$

which is a Junge fit with $\alpha=e^{a}$ and $\beta=-(b+1)$. This results in a clearly improved fit over the length of the CLASP spectra, with a mean value of $\beta$ over the SEASAW runs of 3.15. However, due to the characteristic shape of the SEASAW spectra, this may still result in an overestimation of $\bar{N}$ by over an order of magnitude for small and large values of $r$, with an order of magnitude or more underestimation of $\bar{N}$ for values of $r$ in the middle of the spectrum. Clearly this in not an ideal situation.

Also shown in Fig. 3 are two further functional fits for $\mathrm{d} N / \mathrm{d} r$ which offer an improvement over the simple loglinear Junge fits. The red line shows a quadratic fit of the form $\mathrm{d} N / \mathrm{d} r=\exp \left\{a r^{2}+b r+c\right\}$, while the magenta line shows a piecewise continuous Junge-type fit, with different values of $\beta$ for channels $1-4$ and $4-16$. Both of these fits offer a significant improvement over a simple Junge fit. The piecewise Junge fit has the advantage that it can be used directly 

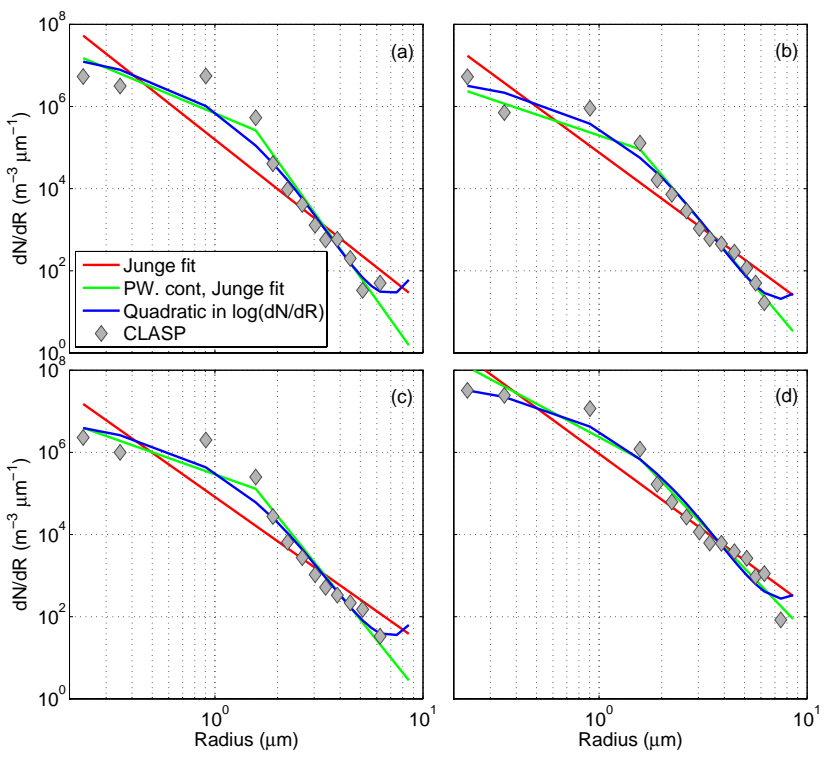

Fig. 3. Four different fits encompassing three different functional forms to aerosol spectra from four randomly chosen 20-min SEASAW runs.

Table 1. Characteristics of $\beta$ values of different Junge-type fits to the SEASAW data

\begin{tabular}{lrrrr}
\hline & Mean & Min & Max & $\sigma$ \\
\hline Junge $\beta$ & 2.708 & 1.756 & 5.188 & 0.440 \\
PW-Junge $\beta_{1}$ & 1.056 & -1.698 & 2.730 & 0.709 \\
PW-Junge $\beta_{2}$ & 5.042 & 3.341 & 6.743 & 0.672 \\
\hline
\end{tabular}

with the F84 and K01 adjustment methods, and will result in different bias velocities for channels $1-4$ and 4-16. The log-quadratic fit has the benefit that it is continuously differentiable and so will result in a unique bias velocity for each CLASP channel, however, it is strictly only valid within the radius range of the CLASP channels, and will diverge quickly from the CLASP recorded spectra outside this range. Using this functional form also complicates the expression for the K01 and F84 bias velocities due to the more complicated derivative with respect to $r$.

The mean error for each CLASP channel resulting from the three functional fits described above are shown in Fig. 4. The left panel shows the absolute error, $\left|N_{i}-F_{i}\right|$, where $F$ is the fit to $N$, and the right panel shows this error normalised by the channel mean number count, $\left|N_{i}-F_{i}\right| / \bar{N}_{i}$. The Junge fit performs reasonably well, but introduces a considerable error at the small and large ends of the CLASP spectrum. This Junge fit does perform better than either of the more complicated functional forms for particle sizes of around $2 \mu \mathrm{m}$, but this is simply where the function changes from underestimating to overestimating the particle count (with increasing $r$ ). The piecewise-linear and log-quadratic fits both perform similarly, and it is not immediately obvious from
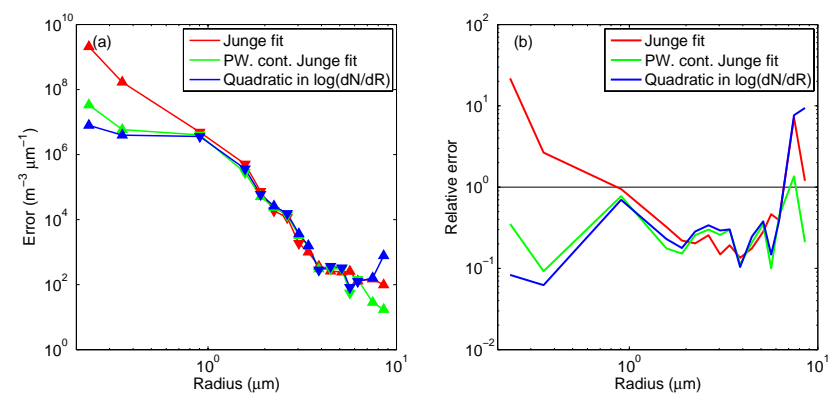

Fig. 4. Mean absolute (a) and relative (b) errors for the fits shown in Fig. 1. The relative errors are simply the absolute error normalised by the mean particle count, $\bar{N}$. The triangles in (a) indicate whether the mean error is positive or negative.

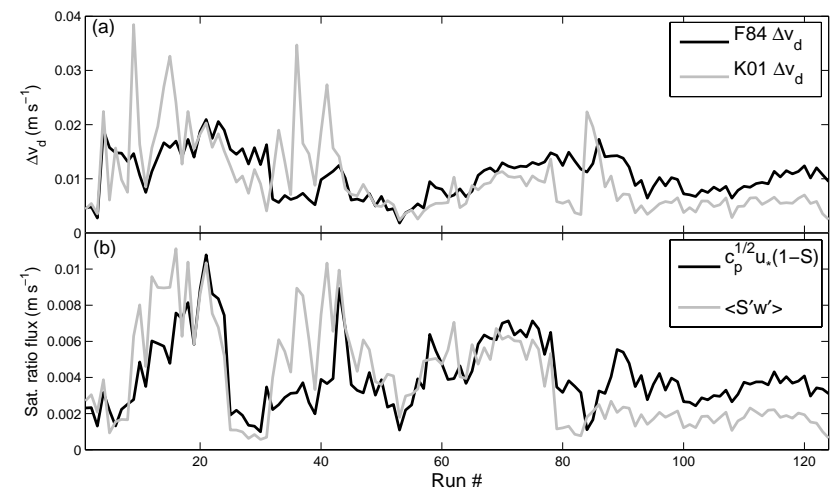

Fig. 5. (a) Run-by-run comparison of $\mathrm{K} 01$ and $\mathrm{F} 84$ methods for calculating bias velocity, $\Delta v_{\mathrm{d}}$; (b) Run-by-run comparison of saturation ratio flux and the F84 approximation to it.

Fig. 4 which provides the best fit. We can calculate this more objectively by summing the relative errors over the CLASP channels. This gives a value of 10.9 for the piecewise-linear Junge fit and 9.2 for the log-quadratic fit, suggesting that the latter provides, on average, a superior fit to the CLASP spectra than does the piecewise-Junge fit. However, as the error metric for both of these fits are similar, we will consider both of these later.

\section{Bulk methods for calculating bias velocity}

The K01 and F84 bias velocities, $\Delta v_{\mathrm{d}}$, given by Eqs. (5) and (7), respectively, both give similar, physically reasonable (Fig. 5a). However, K01's method (mean $=0.010$, $\sigma=0.0069)$ is generally more variable and very slightly smaller than F84's method (mean $=0.011, \sigma=0.0040$ ). Given that the only difference between the K01 and F84 methods is F84's approximation of $\overline{w^{\prime} S^{\prime}}$ as $c_{\mathrm{d}}^{1 / 2} u_{*}(1-S)$, the discrepancy between these methods suggests a significant error in the estimation of the saturation ratio flux. This can be seen more explicitly in Fig. 5b where we have directly compared the saturation ratio flux and F84's approximation to 

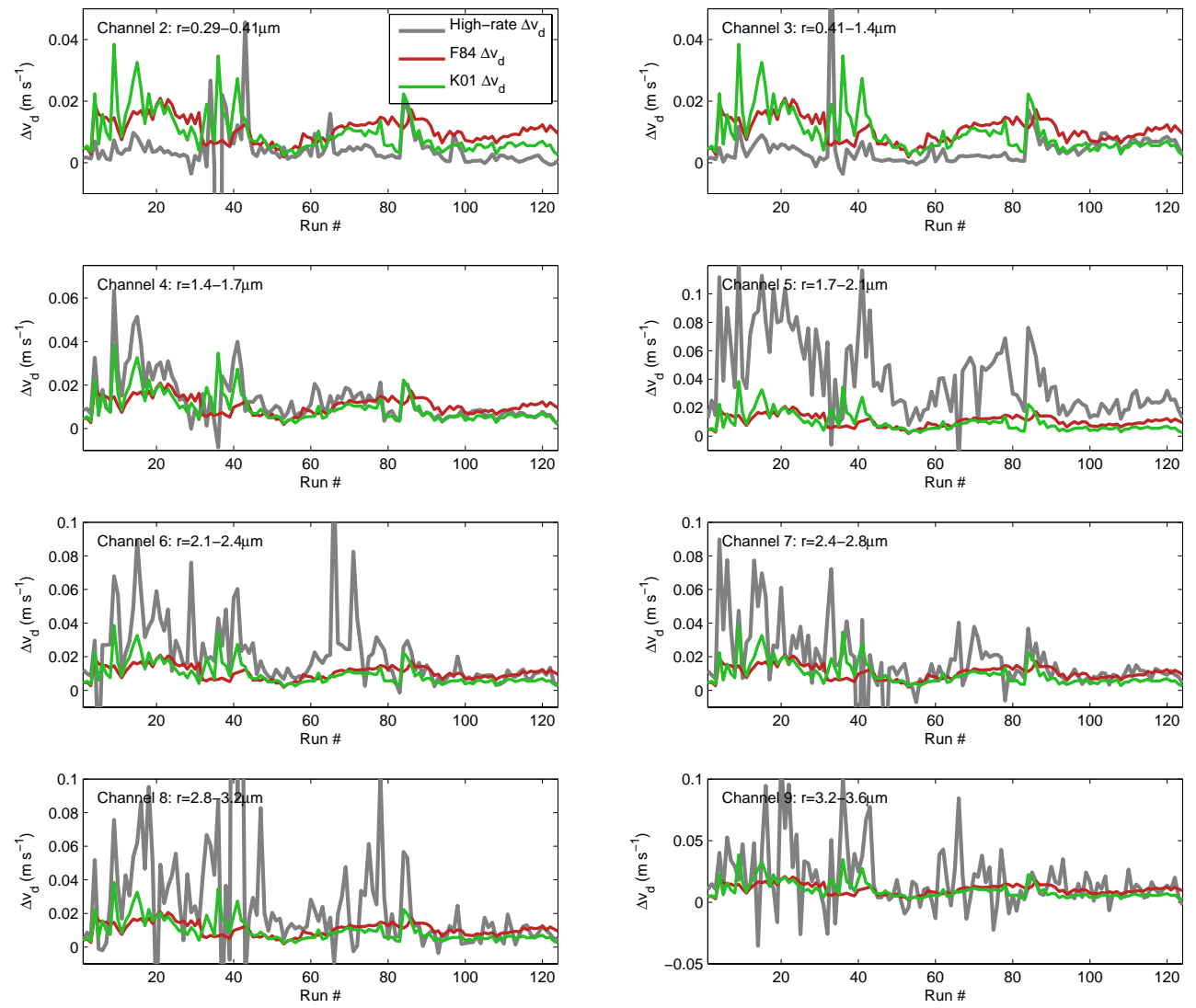

Fig. 6. Apparent deposition velocity due to hygroscopicity, $\Delta v_{\mathrm{d}}$, calculated through K01's and F84's methods, and the difference in the high-rate deposition velocity between ambient and RH-corrected (to run mean) spectra. CLASP channels 2-9 are shown.

it. Where there is a significant saturation flux, F84's method tends to underestimate the saturation ratio flux by up to $50 \%$. Where the saturation ratio is smaller, however, the F84 approximation may overestimate by a similar margin.

\section{Comparison of bulk and high-rate bias estimates}

A comparison of the apparent deposition velocity, $\Delta v_{\mathrm{d}}$, between the K01 and F84 methods and the high-rate method is shown in Fig. 6 for CLASP channels 2-9. Note that in the K01 and F84 methods, $\Delta v_{\mathrm{d}}$ does not depend on particle radius, so only the high-rate $\Delta v_{\mathrm{d}}$ is changing in each of these plots. In channels 2 and 3 the bias velocity from the bulk methods is comparable to that from the high-rate corrections, however, in general they are biased significantly high. This is unsurprising given the assumption that the aerosol spectrum follows a single Junge law (with $\beta \approx 3$ ), whereas, as we saw in Fig. 3, the SEASAW spectra generally have a much shallower gradient than this in channels $1-4$, and a steeper gradient for channels $4-16$. Using $\beta=3$ for the bulk approximations will, thus, inevitably lead to an overestimation of the bias velocity. There are some notable changes in the relationship between bulk and high-rate deposition velocity errors between channels (Fig. 7). These are discussed later.

In CLASP channel 4 , the bulk $\Delta v_{\mathrm{d}}$, particularly from the K01 method, matches extremely closely with that derived from the high-rate method. Generally, it is around channel 4 where the CLASP aerosol spectrum steepens from a gradient of around 1.5 to around 5 (Table 1). Thus, the mean gradient across channel 4 is only slightly larger than 3 so the bulk methods would be expected to perform well here, providing other assumptions and approximations on which these rely are met. Note that the K01 method makes fewer approximations than the F84 method does - namely the use of $\overline{w^{\prime} S^{\prime}}$ rather than an estimate of it - explaining why it generally performs better. This is particularly pronounced in channel 4.

In channels 5-16 the $\beta$-value in the CLASP aerosol spectra increases to typically around 5 , so the assumption that $\beta \approx 3$ again fails, this time resulting in the bulk methods underestimating $\Delta v_{\mathrm{d}}$. This is most pronounced in CLASP channel 5 , although the tendency for the bulk methods to underestimate the bias flux persists through to the larger CLASP channels. As we move to the larger CLASP channels, however, the bias velocity calculated through the high rate method becomes increasingly noisy, making a direct 

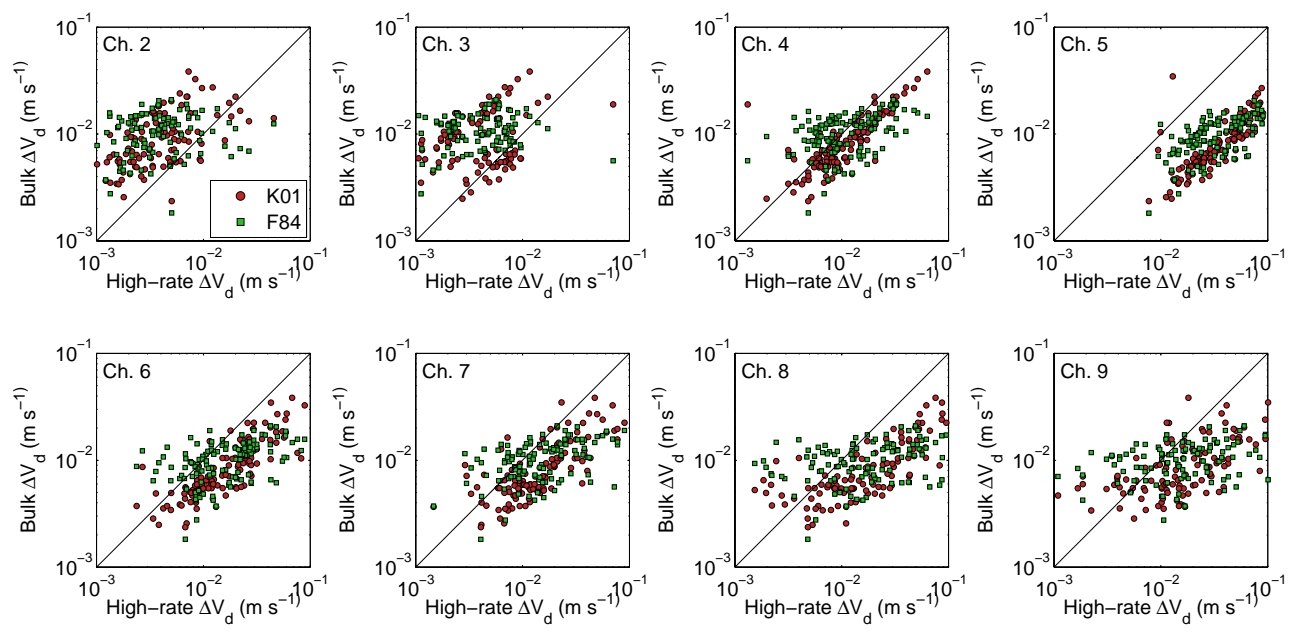

Fig. 7. Scatterplots showing the relationships between CLASP $\Delta v_{\mathrm{d}}$ and K01/F84 $\Delta v_{\mathrm{d}}$ in Fig. 6
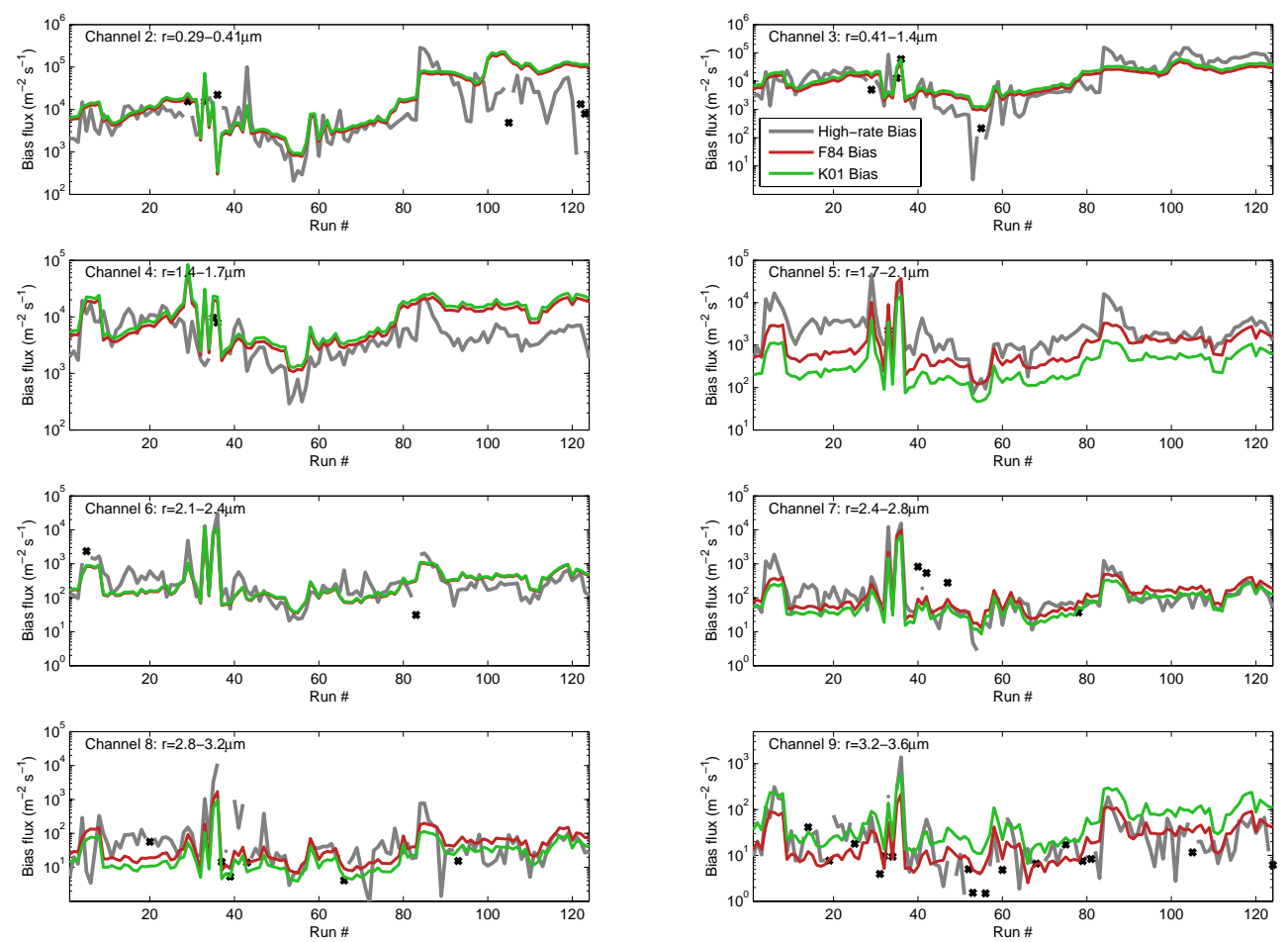

Fig. 8. Flux bias due to hygroscopicity, $\left(=\left.\Delta v_{\mathrm{d}} \bar{N}\right|_{\mathrm{RH}}\right)$, calculated through K01's and F84's methods, and the difference in the high-rate number flux, $\overline{N^{\prime} w^{\prime}}$, between raw and RH-corrected (to run mean) spectra. Black crosses indicate a negative high-rate flux bias. CLASP channels 2-9 are shown.

comparison with the unchanging bulk methods less useful (this is likely due to a decreasing signal-to-noise ratio as fewer and fewer particles are recorded in the larger CLASP channels).

The bias velocity, $\Delta v_{\mathrm{d}}$, is not a true deposition velocity, but an artifact of measuring size-resolved hygroscopic particle concentration in the presence of a humidity flux. However, this apparent deposition will lead to an error in the calculation of the particle flux $\overline{w^{\prime} N^{\prime}}$, by an amount equal to $\Delta v_{\mathrm{d}} \bar{N}_{\overline{\mathrm{RH}}}$, where the $\overline{\mathrm{RH}}$ subscript indicates that $\bar{N}$ has been evaluated at the run mean relative humidity. This bias flux may be comparable to, or larger than, the true particle flux when conditions are close to equilibrium (F84) and, thus, correcting for relative humidity may lead to a change of sign of the particle flux (generally from +ve to -ve when the relative humidity flux is upwards). In general the bias fluxes from 

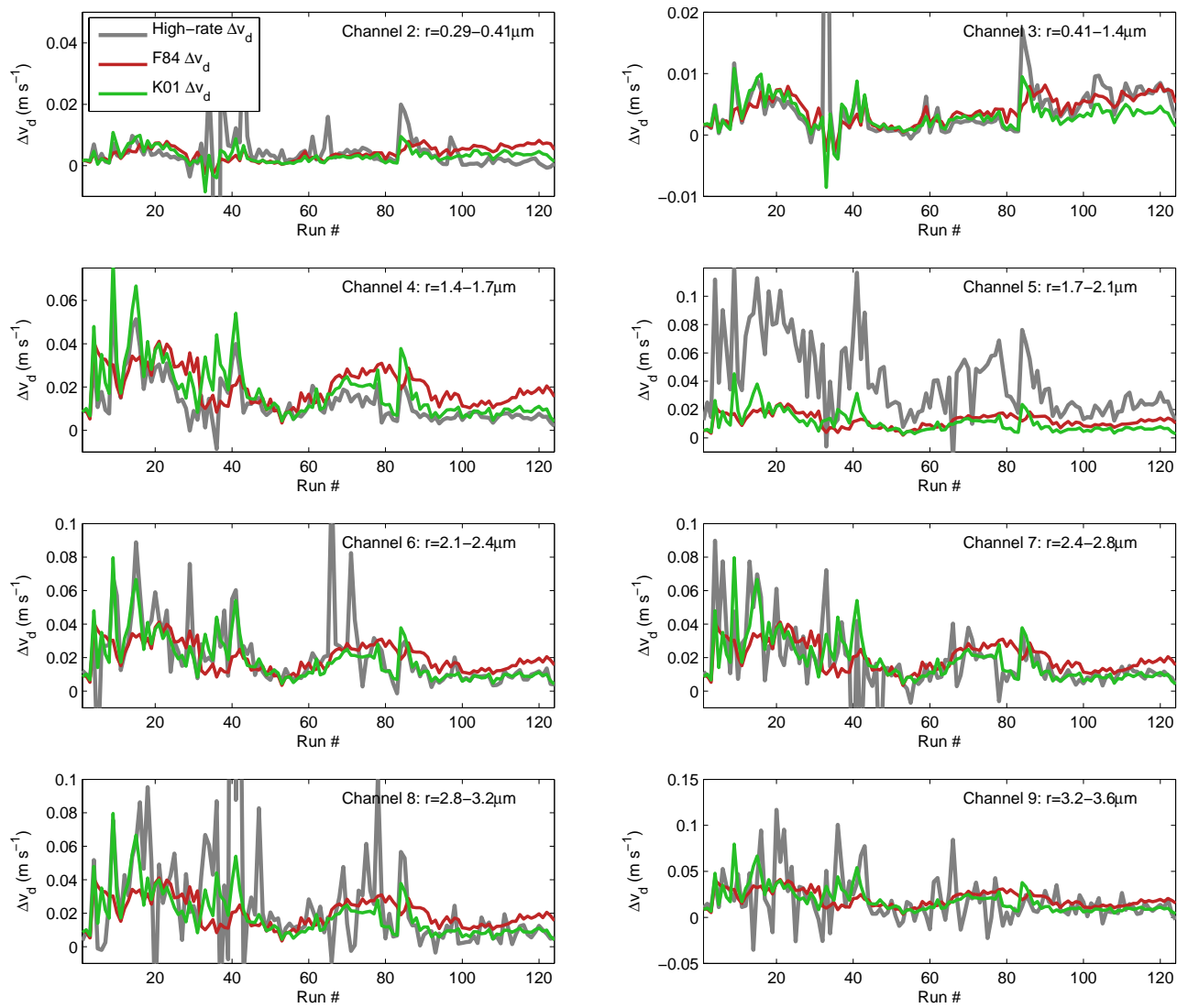

Fig. 9. Apparent deposition velocity due to hygroscopicity, $\Delta v_{\mathrm{d}}$, calculated through K01's and F84's methods, modified to use a piecewise (log-)linear Junge form, and the difference in high-rate deposition velocity between raw and RH-corrected (to run mean) spectra. CLASP channels 2-9 are shown.

the bulk method, particularly from the K01 method, match the high-rate method well (Fig. 8). Where they are in error, they are generally biased high; this is particularly clear in CLASP channel 2 from around run 85 onwards, roughly corresponding to where the saturation ratio flux becomes small. There are also times when the high-rate flux bias is negative, which the bulk methods cannot reproduce for observed values of $\beta$. Note that a negative flux bias may occur in a positive humidity flux as a result of the shape of the aerosol spectrum. If the spectrum is relatively flat for small values of $r$, then the expansion of the channel limits under increasing humidity may cause a loss of particles for certain size channels, even when adjusting to higher relative humidities. A negative bias flux may also result if the spectral shape or mean number concentration is correlated with relative humidity. This may occur as fresh plumes of sea spray (with $N^{\prime}>0$ ) increase the local ambient relative humidity through droplet evaporation. Such a situation would also invalidate the assumption of F84 and $\mathrm{K} 01$ that the dry aerosol distribution is well mixed and uncorrelated with relative humidity.

\subsection{Bulk methods based on improved functional forms}

\subsubsection{A piecewise linear Junge power law}

Given the characteristic shape of the aerosol spectra in the SEASAW dataset, the use of a single Junge power law to approximate the spectra, as suggested by F84 and K01 is not particularly appropriate. An obvious improvement that can be made to this is to use two Junge-type fits: one between channels 1 and 4 where the CLASP spectra are generally quite shallow, and another between channels 4 and 16 where the spectra steepen, modified so these fits produce one piecewise-continuous fit. This gives us different values for $\beta$ for channels 1-3 and 5-16 which can then be used in the F84 and K01 expressions for the bias velocity. Due to the change of gradient at channel $4, \beta$ is undefined here, so we must use a mean of the two $\beta$ values on either side. Generally this will give a value of $\beta \approx 3$ at channel 4 . Examples of this type of fit are shown overlying CLASP spectra in Fig. 3.

\subsubsection{Quadratic fit in $\log (\mathrm{d} N / \mathrm{d} r), r$}

Another approach to dealing with the non-constant (in log space) gradient of the CLASP aerosol spectra is to fit a 

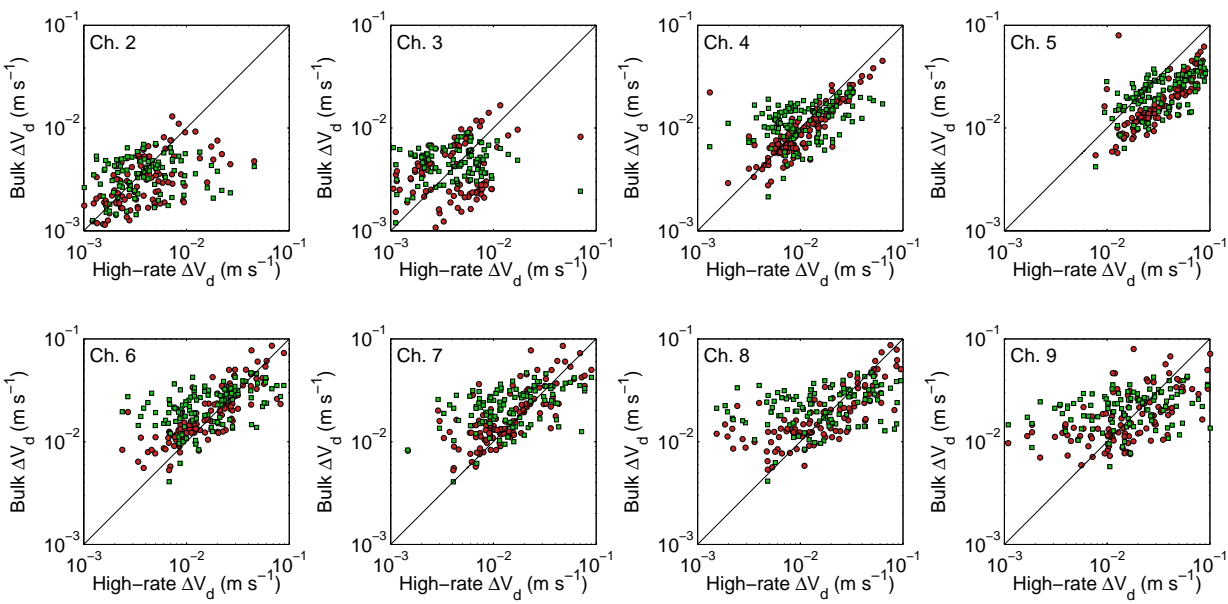

Fig. 10. Scatterplots showing the relationships between the high-rate $\Delta v_{\mathrm{d}}$ and modified K01 (red) and F84 (green) $\Delta v_{\mathrm{d}}$ in Fig. 9 .
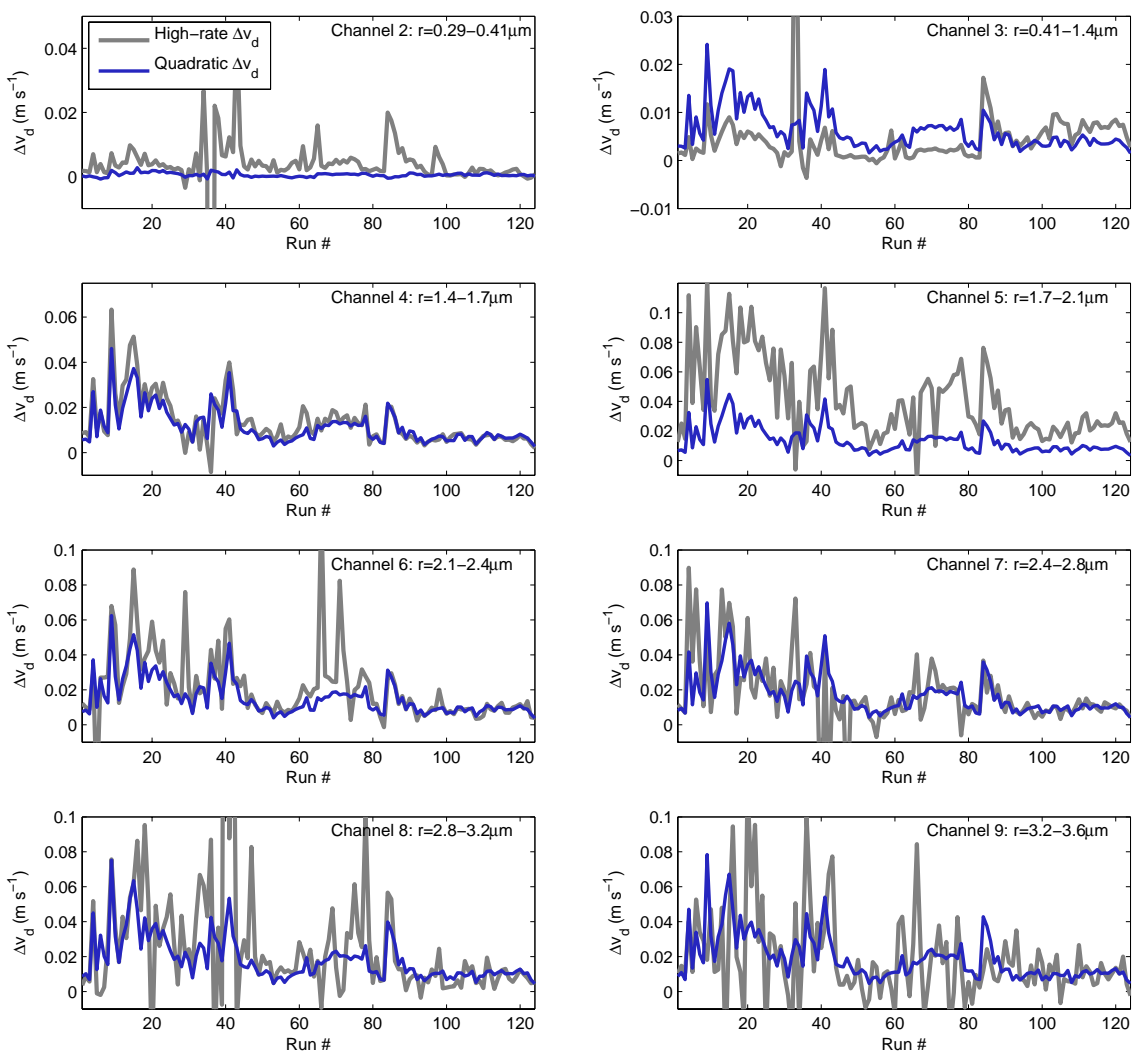

Fig. 11. Apparent deposition velocity due to hygroscopicity, $\Delta v_{\mathrm{d}}$, calculated through K01's method, modified to use a log-linear quadratic functional form, and the difference in high-rate deposition velocity between raw and RH-corrected (to run mean) spectra. CLASP channels 2-9 are shown.

function which is continuously dependent on particle radius. For example, if we fit a quadratic in $\log (\mathrm{d} N / \mathrm{d} r), r$ space, then we end up with a functional representation of the aerosol spectrum of the form

$\frac{\mathrm{d} N}{\mathrm{~d} r}=\exp \left\{a \bar{r}^{2}+b \bar{r}+c\right\}$
Again, examples of this fit are shown along with CLASP measured spectra in Fig. 3. Following K01, we may then derive a bias velocity which is continuously dependent on particle radius, $\bar{r}$. This gives us

$\Delta v_{\mathrm{d}}=\frac{\gamma\left(2 a \bar{r}^{2}+b \bar{r}+1\right)}{3(1-\bar{S})(1-\bar{S}+\gamma)} \overline{w^{\prime} S^{\prime}}$ 

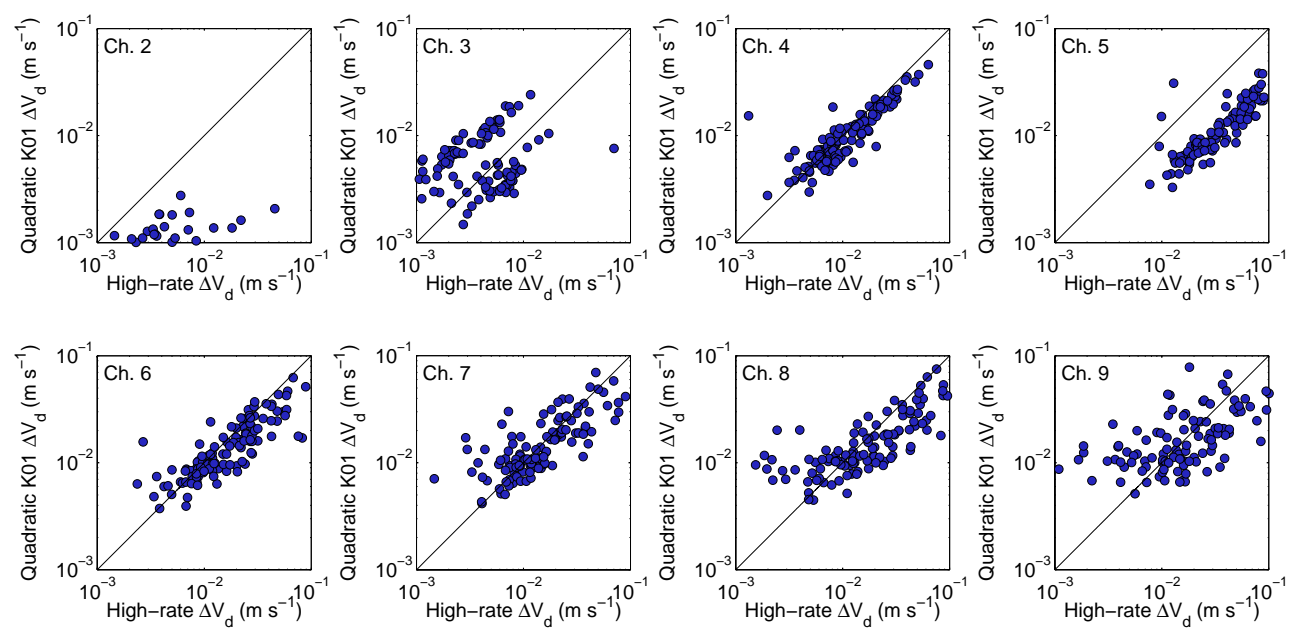

Fig. 12. Scatterplots showing the relationships between high-rate $\Delta v_{\mathrm{d}}$ and K01 (modified to use a log-linear quadratic fit) $\Delta v_{\mathrm{d}}$ in Fig. 11 .
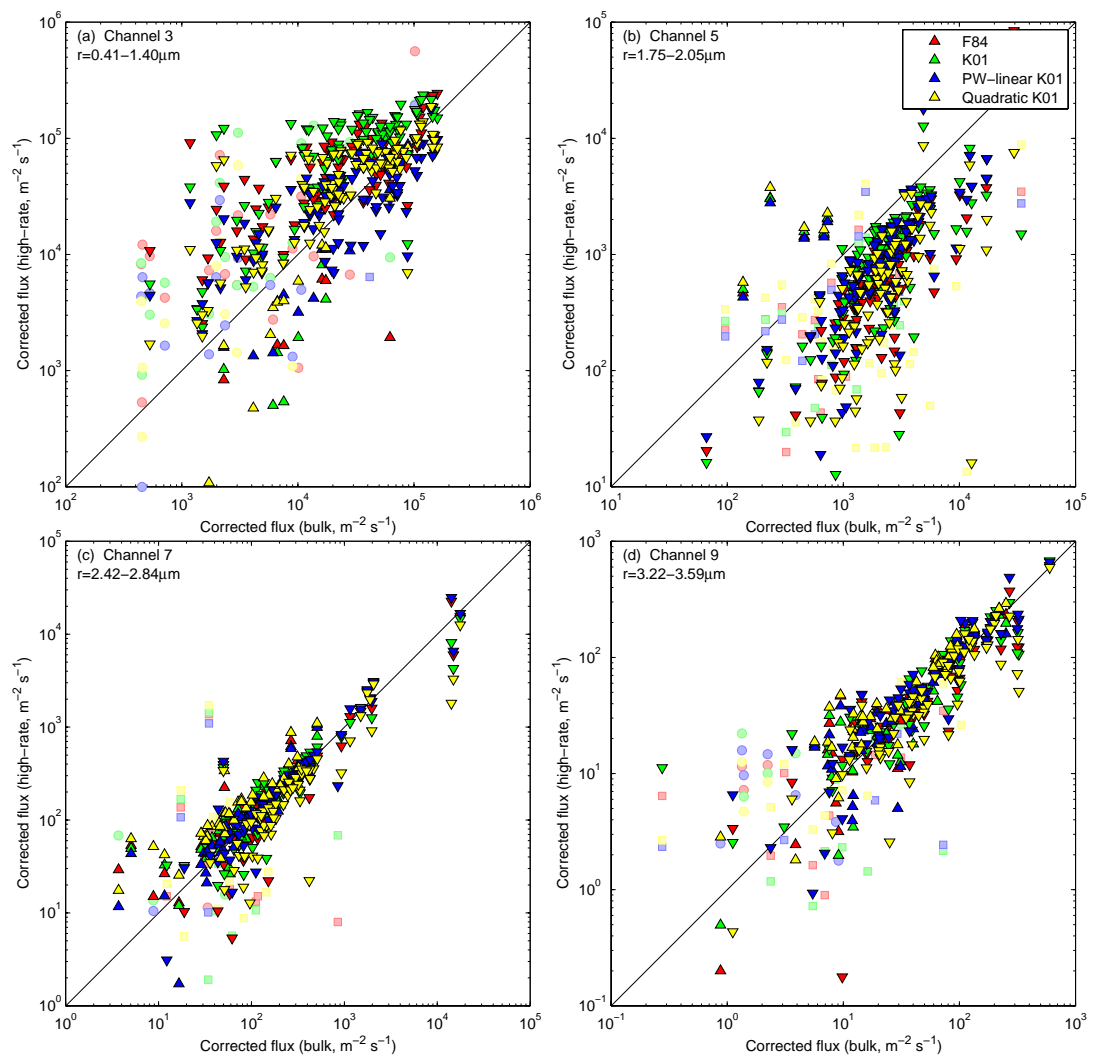

Fig. 13. Comparisons of the high-rate and bulk corrected fluxes for CLASP channels 3, 5, 7 and 9. Upward and downward pointing triangles show where both the high-rate and bulk fluxes are positive or negative, respectively. The lighter points show fluxes of different sign, for positive (circle) and negative (square) high-rate fluxes.

\subsection{Do these adjusted methods offer an improvement?}

Timeseries and scatter-plots showing the relationship between the high-rate corrections and the piecewise-Junge F84 and K01 methods are shown in Figs. 9 and 10, respectively. Using the piecewise continuous Junge fits in place of a single Junge fit is essentially equivalent to multiplying $\Delta v_{\mathrm{d}}$ from the standard F84 and K01 methods by around 0.5 in channels $1-3$ (as the $\beta$ value for a Junge fit over channels $1-4$ is typically around 1.5 ) and by around $5 / 3$ in channels $5-16$ (where the typical $\beta$ value is around 5). Channel 4 , where we must use the mean of the $\beta$ values on each side, generally remains largely unchanged from the standard F84 and K01 estimations of $\Delta v_{\mathrm{d}}$, using $\beta \approx 3$. This scaling has the 
effect of tempering the usual overestimation of $\Delta v_{\mathrm{d}}$ in channels $1-3$ and the underestimation in channels 5-16. However, despite the piecewise continuous Junge fits producing a generally very good approximation of the SEASAW aerosol spectra, there are still times when there are significant differences between the bulk and high-rate methods, suggesting that variables not considered within the bulk methods may be important.

Using K01's method, modified to use a quadratic fit to the CLASP aerosol spectra (Figs. 11 and 12), offers a similar improvement to the estimation of the bias velocity over the standard K01 and F84 bulk methods, particularly at the smaller end of the spectra where the quadratic fit can represent the complete flattening of the spectra which is sometimes observed in the SEASAW aerosol data. The two distinct groupings visible in channel 3 are related to a change in meteorological conditions (particularly the humidity flux) from around run 84 onwards. Before this time, the bulk method tends to overestimate the deposition velocity error and after this time the deposition velocity error is underestimated. This is also apparent, albeit to a much lesser extent and in the opposite sense in channels 4 and 5. This difference is exaggerated in channel 3 because this channel is much wider (in a logarithmic sense) than any of the other channels (see, for example, Fig. 1). The bias apparent in channel 5 is mainly due to a consistent underestimation of the slope of the aerosol spectra by the quadratic fit. It is clear from Fig. 3 that the gradients of the measured spectra tend to increase significantly in gradient between channels 4 and 5 . Such a sharp increase in gradient tends to be underestimated by the functional fit to the spectra (particularly in the case of the smoothly varying quadratic form considered here). The bulk estimates are linearly dependent on the gradient of the aerosol spectra, thus, this underestimation of the gradient leads to a systematic bias in the deposition velocity error. At larger particle sizes, counting statistics become poor and the eddy covariance method becomes an increasingly less valid method of determining aerosol fluxes. This is reflected by the increasingly poor correlations in the larger channels, particularly in channel 9.

Although both the piecewise-Junge and quadratic forms of the K01 correction generally allow an improved bulk representation of the bias velocity (and resulting bias flux) it is clear that no one method is superior under all circumstances seen in the SEASAW data; indeed there are times when the simple Junge fit presented by K01 offers the best approximation to the "true" bias velocity calculated through our high-rate method. Furthermore, there are times when none of the bulk methods considered here provide a suitable approximation of the hygroscopicity-induced bias suggested by the high-rate method. This can be clearly seen in Fig. 13, where comparisons of the particle number fluxes, $\overline{N^{\prime} w^{\prime}}$, corrected using our high-rate method and using the various bulk methods discussed here are shown for CLASP channels 3, 5, 7 and 9.

\section{Conclusions}

The use of the eddy covariance technique to measure the size-segregated flux of sea-spray aerosol (or other hygroscopic aerosol) in the presence of a relative humidity flux may lead to a significant systematic bias in the recorded flux. "Bulk" methods (F84 \& K01) have been presented to account for this bias using mean meteorological conditions (F84) or turbulent measurements (K01) and an assumed mean shape of the aerosol spectra, in the form of a Junge power law.

In this paper, we have developed a method for correcting aerosol spectra for variations in relative humidity at the high temporal resolution required for the calculation of eddy covariance fluxes, allowing the flux bias caused by the relative humidity flux to be explicitly calculated. We have also reformulated the corrections given by F84 and K01 to use a more representative shape of the mean aerosol spectra. In situations where turbulent (high-rate) measurements of humidity are not available and aerosol spectra have not been dried, the bulk correction described by F84 provides a reasonable estimation of the flux correction which must be applied to account for the effects of hygroscopicity. If turbulent measurements of humidity are available, then the bulk correction of K01 offers an improvement, particularly when modified to better model the shape of the mean aerosol spectra. However, these bulk methods are far from infallible and may, at times, significantly under or overestimate the required flux correction. In situations where collocated turbulent aerosol and humidity measurements are available, the high-rate correction method is recommended, despite the relatively high computational cost.

Acknowledgements. SEASAW was funded by the UK Natural Environment Research Council, grant number NE/C001842/1 as part of UK-SOLAS. DAJS was funded by NERC grant NE/H004238/1. We would like to thank two anonymous reviewers whose comments have helped improve this manuscript.

Edited by: A. Stoffelen

\section{References}

Andreas, E. L.: Sea Spray and the Turbulent Air-Sea Heat Fluxes, J. Geophys. Res., 97, 11429-11441, 1992.

Andreas, E. L.: A new sea spray generation function for wind speeds up to $32 \mathrm{~m} \mathrm{~s}^{-1}$, J. Phys. Oceanogr., 28, 2175-2184, 1998.

Andreas, E. L.: A review of the sea spray generation function for the open ocean, in: Atmosphere-Ocean Interactions, edited by: Perrie, W., Vol. 1, 1-46, WIT Press, 2002.

Andreas, E. L., Jones, K. F., and Fairall, C. W.: Production velocity of sea spray droplets, J. Geophys. Res., 115, C12065, doi:10.1029/2010JC006458, 2010.

Brooks, I. M., Yelland, M. J., Upstill-Goddard, R. C., Nightingale, P. D., Archer, S., d'Asaro, E., Beale, R., Beatty, C., Blomquist, B., Bloom, A. A., Brooks, B. J., Cluderay, J., Coles, D., Dacey, J., 
DeGrandpre, M., Dixon, J., Drennan, W. M., Gabriele, J., Goldson, L., Hardman-Mountford, N., Hill, M. K., Horn, M., Hsueh, P.-C., Huebert, B., de Leeuw, G., Leighton, T. G., Liddicoat, M., Lingard, J. J. N., McNeil, C., McQuaid, J. B., Moat, B. I., Moore, G., Neill, C., Norris, S. J., O’Doherty, S., Pascal, R. W., Prytherch, J., Rebozo, M., Sahlee, E., Salter, M., Schuster, U., Skjelvan, I., Slagter, H., Smith, M. H., Smith, P. D., Srokosz, M., Stephens, J. A., Taylor, P. K., Telszewski, M., Walsh, R., Ward, B., Woolf, D. K., Young, D., and Zemmelink, H.: Physical Exchanges at the Air-Sea Interface: Field Measurements from UKSOLAS, B. Am. Meteorol. Soc., 90, 629-644, 2009.

Clarke, A. D., Owens, S. R., and Zhou, J.: An ultra fine sea-salt flux from breaking waves: Implications for cloud condensation nuclei in the remote marine atmosphere, J. Geophys Res., 111, D06202, doi:10.1029/2005JD006565, 2006.

de Leeuw, D., Andreas, E. L., Anguelova, M. D., Fairall, C. W., Lewis, E. R., O’Down, C., Schulz, M., and Schwartz, S. E.: Production flux of sea spray aerosol, Rev. Geophys., 49, RG2001, doi:10.1029/2010RG000349, 2011.

de Leeuw, G., Moerman, M., Cohen, L., Brooks, B., Smith, M., and Vignati, E.: Aerosols, bubbles and sea spray production studies during the RED experiments, in: AMS conference, 2003.

de Leeuw, G., Moerman, M., Zappa, C. J., McGillis, W. R., Norris, S. J., and Smith, M. H.: Eddy Correlation Measurements of Sea Spray Aerosol Fluxes, in: Transport at the Air Sea Interface, edited by: Garbe, C. S., Handler, R. A., and Jähne, B., SpringerVerlag, 2007.

Doss-Hammel, S. M., Zeisse, C. R., Barrios, A. E., de Leeuw, G., Moerman, M., de Jong, A. N., Frederickson, P. A., and Davidson, K. L.: Low-altitude Infrared Propagation in a Coastal Zone: Refraction and Scattering, Appl. Optics, 41, 3706-3724, 2002.

Fairall, C. W.: Interpretation of eddy-correlation measurements of particulate deposition and aerosol flux, Atmos. Environ., 18, 1329-1337, 1984.

Fairall, C. W. and Larsen, S. E.: Dry deposition, surface production and dynamics of aerosols in the marine boundary layer, Atmos. Environ., 18, 69-77, 1984.

Fairall, C. W., Davidson, K. L., and Schaucher, G. E.: An analysis of the surface production of sea-salt aerosol, Tellus B, 35, 31-39, 1983.

Fairall, C. W., White, A. B., Edson, J. B., and Hare, J. E.: Integrated shipboard measurements of the marine boundary layer, Atmos. Ocean. Tech., 14, 338-359, 1997.

Geever, M. C., O’Dowd, D., van Ekeren, S., Flanagan, R., Nilsson, E. D., de Leeuw, G., and Ranmik, Ü.: Submicron sea spray fluxes, Geophys. Res. Lett., 32, L15810, doi:10.1029/2005GL023081, 2005.

Haywood, J. M., Ramaswamy, V., and Soden, B. J.: Tropospheric aerosol climate forcing in clear-sky satellite observations over the oceans, Science, 283, 1299-1393, 1999.

Hill, M. K., Brooks, B. J., Norris, S. J., Smith, M. H., Brooks, I. M., de Leeuw, G., and Lingard, J. J. N.: A Compact Lightweight Aerosol Spectrometer Probe (CLASP), J. Atmos. Oceanic Tech., 25, 1996-2006, doi:10.1175/2008JTECHA1051.1, 2008.

Hoppel, W. A., Frick, G. M., and Fitzgerald, J. W.: The surface source function for sea-salt aerosol and aerosol dry deposition to the ocean surface, J. Geophys. Res., 107, 4382-4399, 2002.
Junge, C. E.: Air Chemistry and Radioactivity, Academic Press, New York, 1963.

Kowalski, A. S.: Deliquescence induces eddy covariance and estimable dry deposition errors, Atmos. Environ., 35, 4843-4851, doi:10.1016/S1352-2310(01)00270-9, 2001.

Large, W. G. and Pond, S.: Open ocean momentum flux measurements in moderate to strong winds, J. Phys. Oceanogr., 11, 324336, 1981.

Lewis, E. R. and Schwartz, S. E.: Comment on "Size distribution of sea-salt emissions as a function of relative humidity", Atmos. Environ., 40, 588-590, 2003.

Mårtensson, E. M., Nilsson, E. D., de Leeuw, G., Cohen, L. H., and Hansson, H. C.: Laboratory simulations and parameterization of the primary marine aerosol production, J. Geophys. Res., 108, 4927, doi:10.1029/2002JD002263, 2003.

Monahan, E. C., Spiel, D. E., and Davidson, K. L.: Whitecap aerosol productivity deduced from simulation tank measurements, J. Geophys. Res., 87, 8898-8904, 1982.

Monahan, E. C., Spiel, D. E., and Davidson, K. L.: A model of marine aerosol generation via whitecaps and wave disruption, in: Oceanic Whitecaps, edited by: Monahan, E. C. and Niocaill, G. M., 167-174, D. Reidel Publishing Company, 1986.

Nilsson, E. D., Ranmik, Ü., Swietliki, E., Leck, C., Aalto, P. P., Zhou, J., and Norman, M.: Turbulent aerosol fluxes over the Arctic Ocean: 2. Wind driven sources from the sea, J. Geophys. Res., 106, 32139-32154, 2001.

Norris, S. J., Brooks, I. M., de Leeuw, G., Smith, M. H., Moerman, M., and Lingard, J. J. N.: Eddy covariance measurements of sea spray particles over the Atlantic Ocean, Atmos. Chem. Phys., 8, 555-563, doi:10.5194/acp-8-555-2008, 2008.

Norris, S. J., Brooks, I. M., Hill, M. K., Brooks, B. J., Smith, M. H., and Sproson, D. A. J.: Eddy Covariance Measurements of the Sea Spray Aerosol Flux over the Open Ocean, J. Geophys. Res., 117, D07210, doi:10.1029/2011JD016549, 2012.

Petelski, T. and Piskozub, J.: Vertical coarse aerosol fluxes in the atmospheric surface layer over the North Polar Waters of the Atlantic, J. Geophys. Res., 111, C06039, doi:10.1029/2005JC003295, 2006.

Reid, J. S., Jonsson, H. H., Smith, M. H., and Smirnov, A.: Evolution of te Vertical Profile and Flux of Large Sea-Salt Particles in a Coastal Zone, J. Geophys. Res., 106, 12039-12053, 2001.

Smith, M. H., Park, P. M., and Consterdine, I. E.: Marine aerosol concentrations and estimated fluxes over the ocean, Q. J. Roy. Meteorol. Soc., 115, 809-824, 1993.

Vignati, E., de Leeuw, G., and Berkowicz, R.: Modeling coastal aerosol transport and effects of surf-produced aerosols on processes in the marine atmospheric boundary layer, J. Geophys. Res., 106, 20225-20238, 2001.

Yelland, M. J., Moat, B. I., Taylor, P. K., Pascal, R. W., Hutchings, J., and Cornell, V. C.: Wind stress measurements from the open ocean corrected for airflow distortion by the ship, J. Phys. Oceanogr., 28, 1511-1526, 1998.

Zhang, K. M., Knipping, E. M., Wexler, A. S., Bhave, P. V., and Tonnesen, G. S.: Reply to comment on "Size distribution of sea-salt emissions and a function of relative humidity", Atmos. Environ., 40, 591-592, 2006. 